\title{
ITCH: THE GOOD, THE BAD AND THE UGLY
}

\section{Distinguished Professor E. Carstens}

\author{
Department of Neurobiology, Physiology and Behavior, University of California, \\ Davis, Davis, CA 95616 USA, *eecarstens@ucdavis.edu
}

\begin{abstract}
Itch (pruritus) is an unpleasant sensation associated with the desire to scratch. The good: a useful purpose of itch is to warn that insects, itchy plants or parasites have invaded the skin, and scratching removes them. Itchy allergic reactions warn to avoid the offending food or skin contact. Scratching to relieve itch is often pleasurable, possibly because scratching excites nerves that convey "pleasant touch". The less good: Like yawning, itch is contagious: watching someone suffering from itch elicits sensations of itch in the viewer. Interestingly, contagious itch activates the same brain areas as itch evoked by a histamine skin-prick test. The bad: Persistent itch often accompanies skin conditions such as eczema, or liver, kidney and other diseases, and represents a major medical and socioeconomic burden worldwide. Chronic itch does not serve a useful purpose and reduces the quality of life, sometimes to the point of suicide. Chronic itch is very poorly treated; antihistamines are usually ineffective, and there is an unmet for improved therapy. The ugly: In extreme cases, neurological disorders cause itch and excessive scratching that damages the skin. A remarkable patient with post-herpetic itch scratched through her skull to expose the brain. In this case, the pain normally evoked by scratching was absent and thus unable to halt further scratching. A positive note: Recent scientific advances are uncovering mechanisms by which receptors in the skin detect itchy substances, and how itch is relayed to the brain, thus identifying targets for improved treatments that reduce or block abnormal itch.
\end{abstract}

Keywords: medicine, itch, pruritus, scratching, spinal cord, contagious itch, neuropathic itch, post-herpetic itch

\section{Introduction}

Itch was defined by Samuel Hafenreffer in 1660 as "an unpleasant sensation that elicits the desire or reflex to scratch." [1]. This definition of itch, or pruritus, is still valid today. Itch is a fundamental sensory modality that, like pain, provides an important warning to the organism to remove a potentially damaging stimulus from the skin. This is commonly accomplished by scratching with finger- or toenails, but may also involve biting, rubbing or other motor behaviors. To the author's knowledge, itch and related scratching behaviors are common to all vertebrate species. Invertebrates such as fruit flies or sea slugs may also experience itch, but this is currently not well understood.

While normal acute itch is a useful warning signal, chronic ongoing itch resulting from dermatitis, systemic disease or other maladies serves no useful purpose and instead causes suffering and reduces the quality of life.
Unfortunately, chronic itch is quite common and represents a largely unmet medical challenge in terms of its alleviation. Under extreme conditions, the unrelenting itch can be so severe that patients contemplate or actually attempt suicide. In this review I wish to discuss the beneficial aspects of itch, as well as the "bad" and "ugly" faces of itch that have prompted new research that has started a revolution in our understanding of itch and its management.

\section{The good}

As noted, itch serves a protective function to warn the organism that something such as an insect, plant spicule or invasive parasite is contacting or penetrating into the skin, and triggers scratching and other movements to remove the offending stimulus and relieve the itch. In this sense it is similar to pain, which protects the body tissue from damage caused by "noxious" (potentially or overtly 
damaging) stimuli, usually by withdrawal of the stimulated limb but also by avoidance, escape or other self-protective reactions. Because of the protective nature of itch and pain, they presumably evolved early on due to the survival benefit they conferred. Thus, they are phylogenetically "older" systems. Indeed, itch and pain are detected and conveyed largely by unmyelinated nerve fibers that still play a major role even in highly evolved vertebrates.

Another good aspect of itch is that, because of the medical and socioeconomic costs associated with chronic forms of itch (see below), considerable efforts have been made recently to better understand the biology of itch. This is good because a variety of factors and mechanisms have been identified that provide targets for therapeutic treatment of itch.

An overview of the itch pathway is shown in Fig. 1. Itch is triggered by an itchy stimulus, usually a chemical factor, that activates the peripheral endings of "pruriceptive" (itchsignaling) nerves in the skin. The most common itch mediator is histamine, which is contained within mast cells of the skin. When released, it causes itch, a swelling, and redness of the skin. In addition to histamine, there are many other non-histamine itch mediators, each acting at its own specific molecular receptor that is contained within nerve endings in the skin (upper right text in Fig. 1). These molecular itch receptors represent targets for development of drugs that can reduce itch at its source.

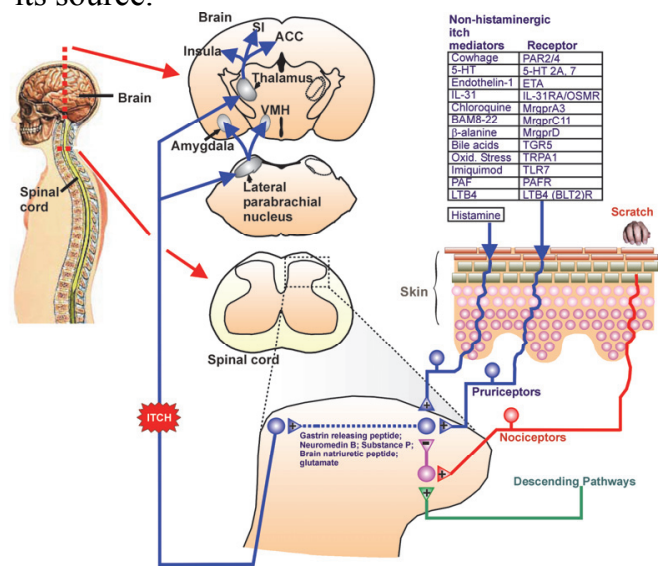

Fig. 1. Itch-signaling pathway (see text).
The itch mediators can be exogenous (from an external source), or may be released from immune cells or damaged skin cells. The itch mediators activate nerves that transmit electrical signals to the spinal cord, where the itch-signaling nerves (pruriceptors) make synapses with neurons. These neurons form a circuit within the spinal cord that utilizes a variety of neuropeptides, as well as the excitatory neurotransmitter glutamate. The spinal neuropeptides and transmitters represent another target to develop drugs that block spinal itch transmission to relieve chronic itch. The spinal itch circuit ultimately activates neurons that give rise to pathways that ascend from the spinal cord to the brain. As shown in Fig. 1 (left side), ascending itch pathways travel to the lateral parabrachial nucleus and thalamus, from which additional neural connections activate a variety of brain areas including the amygdala, insula, somatosensory (S1) cortex and anterior cingulate cortex (ACC), as discussed further below.

Scratching, which is often mildly painful, as well as other painful stimuli such as heat, are well known to inhibit itch. The basis for this lies in a spinal neural circuit comprised of itch-inhibitory interneurons. Painful stimuli activate "nociceptors" (pain-signaling nerves) which in turn activate the spinal inhibitory interneurons (magenta in Fig. 1) that can block the spinal itch signal. Neurophysiological studies have shown that scratching reduces the activity of spinal cord itch-signaling neurons [2]. Spinal inhibition of itch involves wellknown inhibitory neurotransmitters (GABA and glycine) as well as the neuropeptide dynorphin that acts at the $k$-subtype of opiate receptor. This neural circuit provides another target to inhibit itch by increasing spinal inhibition, using drugs such as nalfurafine, which increases inhibition of itch by acting at the $k$ - opiate receptor.

Finally, itch can vary with mood, stress level, and other psychological and physical factors such as temperature. The variability of itch is due, in part, to the actions of neural pathways that descend from the brain to modulate spinal itch transmission (green in Fig. 1), presumably by interacting with itchinhibitory interneurons. This fascinating 
aspect of itch is poorly understood, but may ultimately provide another useful target to develop anti-itch strategies.

\section{Also good}

Most people have experienced that scratching an itch is pleasurable, and subsequent relief of itch is rewarding. It is currently uncertain if the pleasurable effect of scratching an itch is due to the activation of Cfiber low-threshold nerves that are thought to convey a sensation of pleasurable touch [3]. In an interesting study using functional magnetic resonance imaging (FMRI), healthy subjects were subjected to three different conditions: itch alone (no scratching), itch plus scratching of the itchy skin area, or scratching alone (no itch) [4]. They rated pleasantness under each condition. Itch plus scratching was rated as significantly more pleasurable then itch-alone or scratch-alone conditions. Subjects' brains were imaged under each condition. By subtracting images under the scratch alone (not pleasurable) condition from the itch plus scratching (pleasurable) conditions, they identified areas of the brain that responded to the pleasurable aspect. These areas were in the midbrain ventral tegmental area as well as the striatum, regions associated with dopamine and reward. These findings suggest that scratching specifically directed at an itch is pleasurable, and the pleasure may be due to activation of dopaminergic brain reward pathways.

\section{The not-so-good}

A fascinating aspect of itch is that it is socially contagious. Thus, viewing someone who is scratching or exhibiting other signs of itch can elicit sensations of itch, as well as the urge to scratch, in the observer. This is analogous to feeling the urge to yawn when observing someone else yawning. In an interesting study, healthy subjects observed videos of a person scratching or images of itchy skin rashes while undergoing FMRI of their brains. The subjects observing itch exhibited significant activation of brain areas that are also active during physical itch such as following a histamine skin prick. These brain areas include the insula, primary somatosensory cortex and premotor cortex [5].
Activation of the premotor cortex is of interest since this might reflect the urge to scratch. The important message is that when one observes someone else suffering from itch, this activates the observer's brain itch areas to result in itch. It has been suggested that this "empathetic" response might involve a class of brain neurons, called mirror neurons, that respond when someone observes or actually engages in a motor behavior (such as scratching) that they are watching someone else perform.

Contagious itch has also been observed in monkeys and mice [6,7]. Speculatively, this may be of general benefit to social animals by warning of a potentially communicable problem causing itch, such as lice.

\section{The bad}

While acute itch provides a useful warning signal, prolonged chronic itch resulting from dermatitis or other disease serves no useful purpose and instead causes suffering and reduces the quality of life. More than 10 years ago, it was estimated that skin conditions associated with chronic itch affect upwards of $1 / 3$ of Americans and incur socioeconomic costs of approximately $\$ 100$ Billion per year in the US [8]. The costs are undoubtedly higher today. For comparison, it was recently estimated that chronic pain affects 100 million American adults with costs of up to $\$ 635$ billion per year [9]. A recent German study reported a $22 \%$ lifetime prevalence of chronic itch, where chronic itch is defined as lasting $>$ 6 months [10]. Adolescents suffering from chronic itch had a $21.1 \%$ incidence of suicidal ideation, comparable to that of patients suffering from chronic pain [11].

Itch is a dominant feature of a variety of skin conditions including atopic dermatitis (eczema), psoriasis, skin cancer, and burn injury. Moreover, itch is a very frequent symptom of systemic diseases including kidney failure and liver disease. Chronic itch also commonly accompanies a variety of additional pathological conditions such as HIV. Unfortunately, the itch associated with these conditions is poorly managed, and effective treatment of these patients represents an unmet need.

Another negative aspect of chronic itch is the vicious itch-scratch cycle. Thus, itch 
elicits scratching behavior which can lead to skin damage. Damaged skin cells release a variety of inflammatory chemicals which serve to increase, or sensitize, itch. Stronger itch leads to greater scratching, more skin damage, and more itch sensitization, accelerating the vicious cycle.

Antihistamines are ineffective for most types of chronic itch, and there is otherwise a dearth of effective treatments. The silver lining of this dark cloud is that many novel drugs are in varying stages of development to treat chronic itch, so that we can hope for more effective approaches in the near future. Among the promising drugs to combat itch are Substance $\mathrm{P}$ antagonists, $\mu$ - opioid antagonists and $k$-agonists, janus kinase (JAK) antagonists, biologics such as dupilumab, and even botulinum toxin. The rationale for the efficacy of many of these and other drugs in development comes from our recentlyimproved understanding of itch pathways and processing.

\section{The ugly}

Severe chronic itch can occur following herpes infection or injury to peripheral nerves, the spinal cord or brain. Itch following injury to peripheral nerves or the central nervous system is called neuropathic itch.

It has been estimated that $30-65 \%$ of postherpetic patients, and $30 \%$ of patients with peripheral or central nervous system injury, suffer from mild-to-severe itch [12]. This type of itch is again poorly managed, and in some cases can lead to severe injury such as scratch lesions, skin infection etc. A particularly graphic case of neuropathic itch was reported by Oaklander [13]. A 39-year-old woman experienced postherpetic itch on her forehead with no concomitant pain. The itch was so severe that she actually scratched through the front of her skull to expose the brain. When tested, the patient exhibited a severe reduction in pain and touch in the affected area and a $95 \%$ loss of skin innervation. The reduction in pain appears to be a key factor. Normally, scratching activates mechano-sensitive nerve fibers, including those that transmit mechanical pain. The activation of painsignaling neurons excites spinal inhibitory interneurons that suppress itch (Fig. 2A). With a loss of pain-signaling nerves, the ongoing itch increased and elicited enhanced scratching which did not activate pain nerves, such that the inhibitory effect of pain on itch was lost (Fig. 2B). Thus, scratching did no relieve itch, and instead caused further skin damage due the absence of pain.

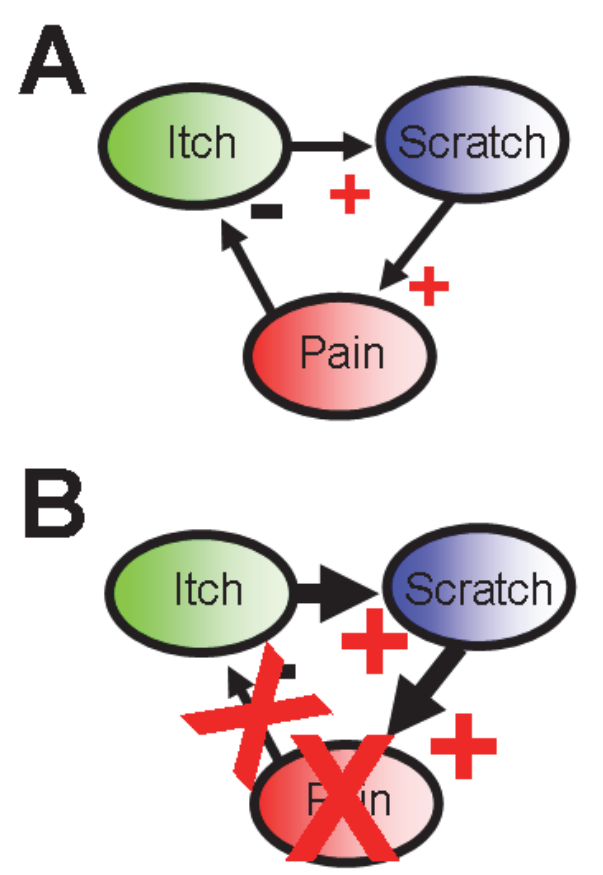

Fig. 2. A: normal condition. Itch elicits scratching that excites $(+)$ pain-signaling neurons that inhibit (-) itch. B: in a neuropathic itch patient, pain nerves are lost. Itchevoked scratching does not cause pain, so itch is no longer inhibited. Itch and scratching thus increase, unabated by pain.

\section{Ending on a good note}

It is largely the bad and ugly aspects of chronic itch that have raised awareness of the magnitude of this problem. This in turn has drawn attention to the biology of itch, a formerly neglected topic that has recently witnessed a revolution, with new research that has prompted major advances in our understanding of normal and pathophysiological mechanisms of itch. This emphasis on the problem of chronic itch provides hope for continued new discoveries that will lead to improved treatments for this all-to-frequently tormenting symptom. 


\section{References}

Hafenreffer, S. Nosodochium, in quo Cutis, Eique Adaerentium Partium, Affectus Omnes, Singulari Method, et Cognoscendi et Curandi Fidelissime Traduntur. Ulm: Kuhn.A (1660).

Akiyama T, Iodi Carstens M, Carstens E. Transmitters and pathways mediating inhibition of spinal itch-signaling neurons by scratching and other counterstimuli. PLoS One. (2011) 6(7): e22665.

Lloyd DM, McGlone FP, Yosipovitch G. Somatosensory pleasure circuit: from skin to brain and back. Exp Dermatol. (2015) 24(5): $321-4$.

Mochizuki H, Tanaka S, Morita T, Wasaka T, Sadato N, Kakigi R. The cerebral representation of scratching-induced pleasantness. J Neurophysiol. (2014) 111 (3): 488-98.

Holle H, Warne K, Seth AK, Critchley HD, Ward J. Neural basis of contagious itch and why some people are more prone to it. Proc Natl Acad Sci U S A. (2012) 109(48): 1981621.

Feneran AN, O'Donnell R, Press A, Yosipovitch G, Cline M, Dugan G, Papoiu AD, Nattkemper LA, Chan YH, Shively CA. Monkey see, monkey do: contagious itch in nonhuman primates. Acta Derm Venereol. (2013) 93(1): 27-9.

Yu YQ, Barry DM, Hao Y, Liu XT, Chen ZF. Molecular and neural basis of contagious itch behavior in mice. Science (2017) 355(6329): 1072-1076.
Bickers DR, Lim HW, Margolis D, Weinstock MA, Goodman C, Faulkner E, Gould C, Gemmen E, Dall T; American Academy of Dermatology Association; Society for Investigative Dermatology. The burden of skin diseases: 2004 a joint project of the American Academy of Dermatology Association and the Society for Investigative Dermatology. J Am Acad Dermatol. (2006) 55(3): 490-500.

Institute of Medicine. Relieving pain in America. A blueprint for transforming prevention, care, education and research. (2011).

Matterne U, Apfelbacher CJ, Loerbroks A, Schwarzer T, Büttner M, Ofenloch R, Diepgen TL, Weisshaar E. Prevalence, correlates and characteristics of chronic pruritus: a population-based cross-sectional study. Acta Derm Venereol. (2011) 91(6): 674-9

Halvorsen JA, Dalgard F, Thoresen M, Bjertness E, Lien L. Itch and pain in adolescents are associated with suicidal ideation: a population-based cross-sectional study. Acta Derm Venereol. (2012) 92(5): 543-6.

Binder A, Koroschetz J, Baron R. Disease mechanisms in neuropathic itch. Nat Clin Pract Neurol. (2008) 4(6): 329-37.

Oaklander AL, Cohen SP, Raju SV. Intractable postherpetic itch and cutaneous deafferentation after facial shingles. Pain. (2002) 96(1-2): 9-12 